PROCEEDINGS OF THE

AMERICAN MATHEMATICAL SOCIETY

Volume 135, Number 10, October 2007, Pages 3273-3282

S 0002-9939(07)09026-0

Article electronically published on June 22, 2007

\title{
NON-ACCUMULATION OF CRITICAL POINTS OF THE POINCARÉ TIME ON HYPERBOLIC POLYCYCLES
}

\author{
PAVAO MARDEŠIĆ AND MARIANA SAAVEDRA \\ (Communicated by Carmen C. Chicone)
}

\begin{abstract}
We call Poincaré time the time associated to the Poincaré (or first return) map of a vector field. In this paper we prove the non-accumulation of isolated critical points of the Poincaré time $T$ on hyperbolic polycycles of polynomial vector fields. The result is obtained by proving that the Poincaré time of a hyperbolic polycycle either has an unbounded principal part or is an almost regular function. The result relies heavily on the proof of Il'yashenko's theorem on non-accumulation of limit cycles on hyperbolic polycycles.
\end{abstract}

\section{INTRODUCTION}

A famous problem of Dulac [2] solved by Écalle [3] and Il'yashenko [6] is the problem of finiteness of the number of limit cycles of a polynomial vector field. The problem reduces to the proof of the non-accumulation of limit cycles on polycycles. The difficulty comes from the non-analyticity of the first return map on the level of a polycycle.

A similar question concerns accumulation of critical points of the Poincaré time on a transversal to a polycycle. We call Poincaré time the time associated to the Poincaré (or first return) map. On periodic orbits it is just the period. The condition of non-criticality of the period appears for instance in the bifurcation theory of subharmonics. Under the non-criticality of the period, zeros of appropriate Melnikov functions guarantee the persistence of a subharmonic periodic orbit of a Hamiltonian under a periodic non-autonomous deformation (see Theorem 4.6.2 of (4)).

The non-accumulation of critical points of the Poincaré time has been proved for bounded polycycles by Chicone and Dumortier in [1] by showing that the time (and its derivative) of passing the corner of any hyperbolic sector tends to infinity. The problem for polycycles all of whose vertices are at infinity is more delicate, because the argument of Chicone-Dumortier is no longer valid in this case. Any polycycle can be desingularized giving a polycyle with only hyperbolic or semi-hyperbolic vertices.

Received by the editors July 5, 2006.

2000 Mathematics Subject Classification. Primary 34C07; Secondary 34C25, 34M35.

Key words and phrases. Critical period, finiteness, non-accumulation, quasi-analyticity, Dulac problem.

This work was partially supported by Fondecyt Projects 1061006 and 7060107, Escuela de Graduados de la Universidad de Concepción and Proyecto Fundación Andes C13955/12. 
In this paper we prove the non-accumulation of critical points of the Poincaré time for a polycyle having only hyperbolic singular points after desingularization. The proof uses geometric ideas from the proof of Il'yashenko's theorem on nonaccumulation of limit cycles on hyperbolic polycyles [5, as well as the second author's result on the asymptotic development of the Poincare time [11.

We believe that the analogy can be pushed further to general polycycles, but the proofs would become much more technical.

\section{Definitions And the MAin Results}

Let $X$ be a polynomial vector field given by

$$
\begin{aligned}
& \frac{d x}{d t}=P(x, y), \\
& \frac{d y}{d t}=Q(x, y),
\end{aligned}
$$

where $P$ and $Q$ are real polynomials. The foliation given by the vector field $X$ can be extended to the Poincaré disc $\mathbb{D}^{2}$ bounded by the line at infinity $\ell_{\infty}$. Consider a polycycle $\Gamma$ of $X$ in $\mathbb{D}$. Given an analytic transversal section $\Sigma$ to the polycycle $\Gamma$ with a local analytic coordinate $s \in[0, \epsilon)$ parameterizing the side of $\Sigma$ where the Poincaré first return map is defined, let $T:(0, \epsilon) \rightarrow \mathbb{R}^{+}$be the corresponding Poincaré time function of the vector field $X$ along $P$.

Theorem 1.1. Let $X$ be a vector field as above and $\Gamma$ a polycycle in the Poincaré disc $\mathbb{D}$ such that only hyperbolic vertices appear in its desingularization. Let $\Sigma$ be any analytic transversal to the polycycle $\Gamma$ parametrized analytically by $[0, \epsilon)$, with 0 corresponding to the polycycle. Let $T:(0, \epsilon) \rightarrow \mathbb{R}^{+}$be the Poincaré time on $\Sigma$. If critical points of the function $T$ accumulate on 0 , then the Poincaré time $T$ is constant.

If the Poincaré time of a vector field along a polycycle $\Gamma$ measured on a given transversal $\Sigma$ is constant, we say that the polycycle is isochronous on $\Sigma$. If the Poincaré map along a polycycle is the identity, the notion of isochronicity is independent of the choice of the transversal. This is no longer the case in general. Isochronicity corresponds to the existence of a transversal foliation invariant with respect to the flow of the vector field.

In order to study the Poincaré time $T$ along a polycycle, one studies the Poincaré time $T$ of the polycycle obtained by desingularization, which we assume hyperbolic.

Let $p_{i}, i=1 \ldots, k$, (with a cyclic order) be the singular points (all hyperbolic) of the desingularized polycycle. Let $\Sigma_{i}$ and $\Pi_{i}$ be analytic transversals to the polycycle near $p_{i}$ in a sufficiently small neighborhood of $p_{i}$. Let $D_{i}$ be the Dulac map from $\Sigma_{i}$ to $\Pi_{i}$ and $T_{i}$ the corresponding time function of the saddle $p_{i}$, which we call Dulac time. Let $R_{i}$ be the regular Poincaré functions from $\Pi_{i}$ to $\Sigma_{i+1}$, with their corresponding time functions $\tau_{i}$ (see Figure 1) for $1 \leq i \leq k-1$ and put $R_{0}=D_{0}=i d$. 


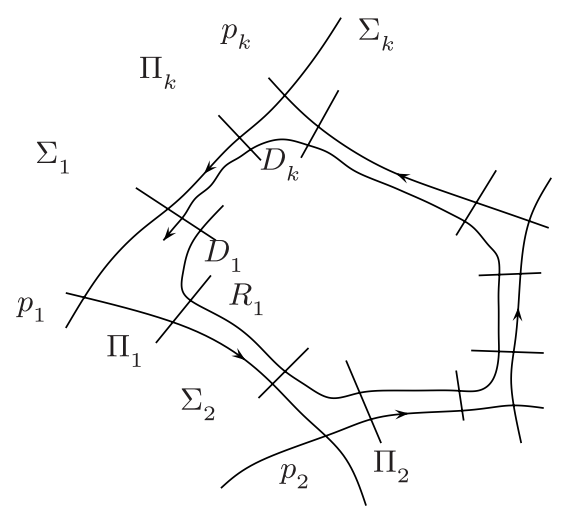

Figure 1. Poincaré map of a polycycle

The Poincaré time $T$ measured at $\Sigma_{1}$ can be written in the form

$$
\begin{aligned}
T & =\sum_{i=0}^{k-1} T_{i+1} \circ\left(R_{i} \circ D_{i}\right) \circ \cdots \circ\left(R_{1} \circ D_{1}\right) \\
& +\sum_{i=0}^{k-1} \tau_{i+1} \circ D_{i+1} \circ\left(R_{i} \circ D_{i}\right) \circ \cdots \circ\left(R_{1} \circ D_{1}\right) .
\end{aligned}
$$

Note that all contributions in (1.1) are non-negative.

An asymptotic development of the Dulac maps $D_{i}$ was given by Dulac in [2]. A similar result for the Poincaré time function $T$ and its derivative $T^{\prime}$ is given by the second author in [11. In order to formulate these results, let us recall the notions of semi-regular germs and semi-regular homeomorphisms.

\section{Definition 1.2.}

(i) A Dulac series is a formal series $\hat{f}$ of the form

$$
\hat{f}(s)=\sum_{k \in \mathbb{N}_{0}} s^{\mu_{k}} P_{k}(\log s), \quad s>0,
$$

where $\left\{\mu_{k}\right\}$ is a strictly increasing unbounded sequence of real numbers and $P_{k}$ is a real polynomial.

(ii) We call a $C^{\infty}$ germ $f$ at 0 in $\mathbb{R}^{+}$a semi-regular germ if it has an asymptotic Dulac series, that is, if for any $N$, there exists a partial sum $S$ of the Dulac series such that $f-S=o\left(x^{N}\right)$.

(iii) A germ $g$ is said to be a semi-regular homeomorphism if it is semi-regular and moreover its asymptotic development (1.2) verifies $\mu_{k}>0$ and $P_{0}$ is a positive constant.

Proposition 1.3 (Dulac [2]; see also [6]).

(i) Dulac maps $D_{i}$ associated to hyperbolic singularities are semi-regular homeomorphisms.

(ii) Semi-regular homeomorphisms form a group with respect to the composition.

Proposition 1.4 (Saavedra 9], [10] and [11]).

(i) Dulac times $T_{i}$ associated to hyperbolic singularities are semi-regular. 
(ii) Let $f$ be semi-regular and let $g$ be a semi-regular homeomorphism. Then the composition $f \circ g$ is semi-regular.

(iii) Semi-regular germs form a real vector space.

(iv) Let $X$ be a polynomial vector field, $\Gamma$ a polycycle and $T$ the Poincaré time function along $\Gamma$ as in Theorem 1.1. Then the function $T$ is semi-regular.

Remark 1.5. In our work, in addition to holomorphic (or meromorphic) functions, we have to work with Dulac maps $D_{i}$ (which are semi-regular homeomorphisms) and the Dulac time maps $T_{i}$ (which are only semi-regular). This is the reason that, contrary to Il'yashenko in [6], we have to distinguish between these two classes. Functions which we call semi-regular homemorphisms are called just semi-regular in [6].

Note also that a composition of two semi-regular germs is not necessarily defined and even if it is defined, it does not necessarily give a semi-regular germ.

Theorem 1.6. Let $X$ be a polynomial vector field, $\Gamma$ a polycycle of $X, \Sigma$ a transversal to the polycle and $T$ the Poincaré time along the polycycle on $\Sigma$, as in Theorem 1.1. If the Poincaré time $T$ of a hyperbolic polycycle has constant asymptotic development (only one term which is a constant), then the Poincaré time is constant.

Theorem 1.6 will be a direct consequence of the following theorem:

Theorem 1.7. Consider the mapping which associates to Poincaré time functions of hyperbolic polycycles their Dulac asymptotic development. Then, the restriction of this mapping to Poincaré times having bounded principal part is injective.

Remark 1.8. Theorem 1.7 is a kind of quasi-analyticity theorem for the Poincaré time analogous to the corresponding theorem of Il'yashenko, for the Dulac map of a hyperbolic polycycle.

The theorem is probabaly true more generally for any Poincaré time of a hyperbolic polycycle, but our proof applies only to Poincaré time functions with bounded principal part.

\section{Almost Regular mappings}

In this section we define almost regular maps and almost regular homeomorphisms. We also recall some general results for almost regular germs. Almost regular functions were introduced by Il'yashenko for studying the Dulac map of a hyperbolic saddle. In the next sections we will prove that the time function of a hyperbolic polycycle is almost regular.

Consider an analytic function $f:(0, \epsilon) \rightarrow \mathbb{R}$ and its extension to the complex domain. As this extension can be multivalued, it is considered in the logarithmic chart. More precisely, in the rest of the paper we denote by $E$ the mapping

$$
E(\zeta)=\exp (-\zeta)
$$

and we consider the analytic extension of the mapping $f \circ E:[0,+\infty) \rightarrow(0,1]$. It is proved by Il'yashenko in [5] that the Dulac map $D$ of a hyperbolic saddle in the logarithmic chart has an analytic extension to a sufficiently big domain $\Omega_{b}$ called a standard quadratic domain (see Figure 2). 

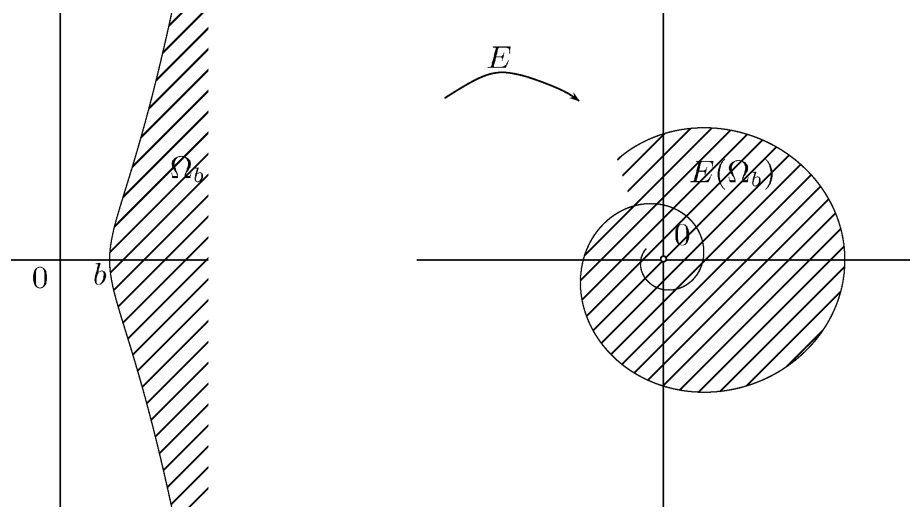

FIGURE 2. Standard quadratic domain and its image by $E$

More precisely, for some $b>0, D \circ E$ has a holomorphic extension to a domain of the form

$$
\Omega_{b}=\left\{\zeta \in \mathbb{C} \mid \operatorname{Re}(\zeta)>b\left(1+(\operatorname{Im}(\zeta))^{2}\right)^{1 / 4}\right\} .
$$

Definition 2.1. A germ $f$ of a real analytic function at $0 \in \mathbb{R}^{+}$is called almost regular if it verifies (i) and (ii):

(i) $f$ is semi-regular,

(ii) the function $f \circ E$, where $E: \zeta \mapsto e^{-\zeta}$ admits a holomorphic extension to a domain of the form $\Omega_{b}$, given by (2.2) for some $b>0$.

An almost regular germ is called an almost regular homeomorphism if all exponents $\mu_{k}$ in its asymptotic development (1.2) are positive and $P_{0}$ is a strictly positive constant.

Using this terminology, Il'yashenko proves

Theorem 2.2. The Dulac map of a hyperbolic saddle is a bounded almost regular homeomorphism.

\section{Proposition 2.3.}

(i) Almost regular homeomorphisms with respect to the composition form a group.

(ii) Let $f$ be almost regular and $g$ an almost regular homeomorphism. Then $f \circ g$ is almost regular.

(iii) Almost regular germs form a real vector space.

Proof. Claim (i) appears in Il'yashenko [6]. Claim (ii) follows using (ii) of Proposition 1.4. Claim (iii) is obvious.

The interest in almost regular mappings lies in the following theorem of Il'yashenko [5] (see also the presentation in 7]).

Proposition 2.4. Let $\tau$ be an almost regular germ such that the extension of $\tau \circ E$ to a standard quadratic domain $\Omega_{b}$ is bounded. Assume moreover that the Dulac series $\hat{\tau}$ of $\tau$ is zero. Then the function $\tau$ is zero.

The theorem is proved by a double application of the Phragmen-Lindelöf theorem: 
Proposition 2.5 (Phragmen-Lindelöf theorem [12]). Let $f$ be a holomorphic function in a closed sector $D$ of width $\pi / \alpha$ at the origin. Assume that for some $\beta<\alpha$,

$$
f(z)=O\left(e^{|z|^{\beta}}\right)
$$

uniformly in the sector, for $|z| \rightarrow \infty$ and let $\sup _{z \in \partial D}|f(z)|<\infty$. Then

$$
\sup _{z \in \partial D}|f(z)|=\sup _{z \in D}|f(z)| .
$$

Proof of Proposition 2.4. Note that $\tau \circ E$ is asymptotically exponentially flat on the positive real axes. Let $\phi: \mathbb{C}^{+} \rightarrow \mathbb{C}$ be given by $\phi(\eta)=b^{\prime}(1+\eta)^{1 / 2}+\eta$. Here $\mathbb{C}^{+}=\{\zeta \mid \operatorname{Re}(\zeta) \geq 0\}$. Then, for $b^{\prime}>0$ sufficiently big, the image $\phi\left(\mathbb{C}^{+}\right)$is contained in the quadratic domain $\Omega_{b}$. The function $f=\tau \circ E \circ \phi$ defined on the positive complex semiplane $\mathbb{C}^{+}$is bounded and exponentially flat on the positive real axes. That is, there exists $K$ such that $|f(\eta)|<K$ for any $\eta \in \mathbb{C}^{+}$, and for any $n \in \mathbb{N}$, there exists $K_{n}$ such that $|f(\operatorname{Re}(\eta))|<K_{n} e^{-n \operatorname{Re}(\eta)}$. Let $f_{n}(\eta)=f(\eta) e^{n \eta}$. Then $\left|f_{n}(\eta)\right|<K e^{n|\eta|}$, so one can apply the Phragmen-Lindelöf theorem to $f_{n}$ on the first quadrant, given that in the first quadrant $\left|f_{n}\right|$ is bounded by a bound on the boundary of the first quadrant, that is, by $\sup \left\{K, K_{n}\right\}$. The same argument applies also to the fourth quadrant. Putting the two quadrants together and applying again the Phragmen-Lindelöf theorem on their union, one gets that $\left|f_{n}\right|$ is bounded by $K$ on $\mathbb{C}^{+}$. Passing to the limit for $n \rightarrow+\infty$, one gets that $f$ vanishes identically on $\mathbb{R}^{+}$and hence also on $\mathbb{C}^{+}$.

Remark 2.6. Note that in the proof of Proposition 2.4 the validity of the asymptotic development is required only for real values of $x$.

\section{The Dulac time function}

In this section we study the analytic extension of the Dulac time function at saddle points obtained after desingularization of singular points at infinity. Note that if a polycycle has at least one vertex at finite distance, then the Dulac series of the Dulac time of the vertex tends to infinity

In local analytic coordinates at infinity, after desingularization, a saddle point is in the following prenormal form:

$$
\begin{aligned}
& \dot{x}=\frac{1}{x^{m} y^{n} g(x, y)} x, \\
& \dot{y}=\frac{1}{x^{m} y^{n} g(x, y)}(-\lambda y(1+h(x, y))),
\end{aligned}
$$

where $m, n$ are integers, $\lambda>0, g$ and $h$ are analytic functions, $g(0,0)>0$ and $|h(x, y)|=O(|x y|)$.

We must allow the factor $\frac{1}{x^{m} y^{n}}$ with $m$ and $n$ possibly positive, as the polycycle we study can be unbounded and in local coordinates at infinity and after desingularization we can get such factors. Of course, when studying time, we have to work with the conjugation relation for vector fields and not just equivalence and hence we must take into account multiplicative factors. 


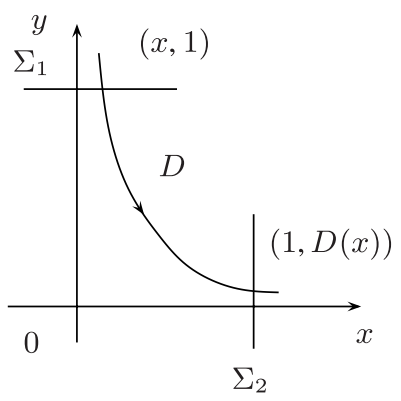

Figure 3. Dulac map

Remark 3.1 (10, [11]).

(i) The principal part of the Dulac time $T_{i}$ of (3.1) is

$$
\begin{aligned}
& c x^{r} \log ^{\beta} \text {, with } c>0, r=\min \{\lambda n, m\}, \beta \in\{0,1\}, \\
& \text { where } \beta=1, \quad \text { if } \lambda n-m=0 .
\end{aligned}
$$

(ii) If the principal part of the Dulac time $T_{i}$ of (3.1) is bounded, then $m \geq 0$, $n \geq 0$ and $(m, n) \neq 0$.

We consider the variables $x, y$, as well as the time $t$, as complex variables. The functions $g$ and $h$ can be assumed holomorphic in the bidisc $\Delta=\Delta_{1} \times \Delta_{1}$, where $\Delta_{1} \subset \mathbb{C}$ is the closed unitary disc.

Let $\Sigma_{1}=\Delta_{1} \times\{1\}$ and $\Sigma_{2}=\{1\} \times \Delta_{1}$ be transversals to the $x$ and $y$ axes and let $D$ be the Dulac map from $\Sigma_{1}$ to $\Sigma_{2}$ (see Figure 3).

Il'yashenko [6] proves that the Dulac map $D$ of the saddle point is an almost regular homeomorphism. He proves in particular that $D \circ E$ extends to a standard quadratic domain $\Omega_{b}$. We use the following formulation, which is the essence of the proof. For any $\zeta \in \mathbb{C}^{+}$, let $K_{\zeta}$ denote the rectangle with vertices $(0,0),(\operatorname{Re}(\zeta), 0), \zeta$, $(0, \operatorname{Im}(\zeta))$.

Theorem 3.2 (Il'yashenko). Let us be given a system of differential equations of the form (3.1). Moreover, assume that the function $h$ is analytic in the bidisk $\Delta$.

Then there exists $b>0$ such that for $\zeta \in \Omega_{b}$ and any path $\gamma_{\zeta} \subset K_{\zeta}$ defined on $[0,1]$ with $\gamma_{\zeta}(0)=\zeta$ and $\gamma_{\zeta}(1)=0$, the path $E \circ \gamma_{\zeta}$ can be lifted to a path $\widetilde{E \circ \gamma}$ in the leaf of the foliation defined by the differential equation (3.1). Moreover, the lift $\widetilde{E \circ \gamma}$ remains within the bidisk $\Delta$ starting at $(E(\zeta), 1) \in \mathbb{C}^{2}$ and ending at $\widetilde{E \circ \gamma}(1)=(1, D \circ E(\zeta))$, with $D$ an analytic (multivalued) extension of the Dulac map to the domain $E\left(\Omega_{b}\right)$.

In fact, in the proof of Il'yashenko's Theorem as given for instance in [8], the existence of a quadratic domain $\Omega_{b}$ with the above properties is proved only for paths $\gamma_{\zeta}$ joining $\zeta$ and 0 obtained by joining the upper and the left side of the rectangle $K_{\zeta}$. The same estimates remain valid for any path $\gamma_{r, \zeta}, 0 \leq r \leq 1$, obtained by joining the two segments $[\zeta, r \operatorname{Re}(\zeta)+i \operatorname{Im}(\zeta)]$ and $[r \operatorname{Re}(\zeta)+i \operatorname{Im}(\zeta), r \operatorname{Re}(\zeta)]$.

Theorem 3.3. Extension of the time function Let us be given a system of differential equations in the form (3.1). Let $h, b, E, \Omega_{b}$, and $\gamma_{\zeta}$ be as above, assume $g$ holomorphic in $\Delta$ and let $T$ be the corresponding Dulac time function. 
(i) Then $T$ is almost regular.

(ii) Assume moreover that the Dulac series of $T$ contains no unbounded terms. Then the function $T \circ E$ is bounded on a whole quadratic domain $\Omega_{b}$ to which $T \circ E$ extends.

Proof. In order to prove claim (i) of the theorem, due to Proposition 1.4 it remains to prove the extendability of $T \circ E$ to a quadratic domain $\Omega_{b}$.

The initial (real) Dulac time function $T$ is calculated in the prenormal coordinates (3.1) by integrating the form

$$
d t=x^{m} y^{n} g(x, y) \frac{d x}{x}
$$

along a solution of the equation (3.1) from $(x, 1)$ until cutting the transversal $x=1$ at $(1, D(x))$.

Considering the logarithmic chart $E$ is just a change of variables in integration. So $T \circ E$ for $E(\zeta)=x$ and $\zeta$ real is given by the integral of $d t$ from $\zeta$ to 0 . The value of the analytic extension of $T \circ E$ at $\zeta$ is obtained by integrating the form $d t$ given in (3.3) along paths $\gamma_{\zeta}$ as given in Theorem 3.2. Moreover, by Theorem 3.2 . for any $\zeta$ belonging to the quadratic domain $\Omega_{b}$ as given in Theorem 3.2. any path $\gamma_{\zeta}$ in $K_{\zeta}$ composed with $E$ lifts to a path tangent to the leaves of the foliation given by (3.1). By the construction of $\Omega_{b}$, this path in the $(x, y)$-space is contained in the bidisc of holomorphy of the function $g$. The polar locus of the form $d t$ is contained in the union of the coordinate axes and the path of integration avoids the axes. Note that any path $\gamma_{\zeta}$ connecting $\zeta$ and 0 and belonging to the rectangle $K_{\zeta}$ gives the same value of the extension of the Dulac time function, as any two such paths are homotopic.

We use the standard theorem that the integral of a continuous family of integrable holomorphic forms on an interval gives a holomorphic function to see that the extension of $T \circ E$ to the quadratic domain $\Omega_{b}$ is analytic.

In order to prove (ii), note that by Remark 3.1 the assumption that the (real) Dulac series is bounded implies that $m \geq 0, n \geq 0,(m, n) \neq(0,0)$. If $m=0$ and $n \geq 1$, then we note that the Dulac time from $y=1$ to $x=1$ is the same as the Dulac time that the opposite field takes to go from $x=1$ to $y=1$. Passing from one to the other involves only a reparametrization by a Dulac map, which is an almost regular homeomorphism. We can hence assume that $m \geq 1$ in (3.3).

To show the boundedness, we calculate the Dulac time directly in the $x$-coordinate viewing the form $d t$ as a multivalued form. The multivaluedness comes from the multivaluedness of $y$ as a function of $x$. We estimate the integral after passing to the polar coordinates in the $x$ plane:

$$
x=r e^{i \theta} .
$$

We choose as path of integration in the $x$ plane the image by $E$ of the path consisting of a horizontal segment from $\zeta$ to the boundary of $\Omega_{b}$, followed by the part of the boundary of $\Omega_{b}$ up to its intersection with the $\operatorname{Re}(\zeta)$ axes and the horizontal segment up to the origin. This path belongs to the rectangle $K_{\zeta}$. The image of this path by $E$ corresponds to a radial part from $x$ to the spiral $E\left(\partial \Omega_{b}\right)$, followed by a part of the spiral $E\left(\partial \Omega_{b}\right)$ ending on the positive real axes and a radial path on the positive real axes up to the point 1 in the $x$ plane (see Figure 4).

Note that as $m \geq 1$, it follows that the function $x^{m-1} y^{n} g(x, y)$ is bounded by some $M>0$, for $(x, y) \in \Delta$. In order to show that the Dulac function is bounded, it 


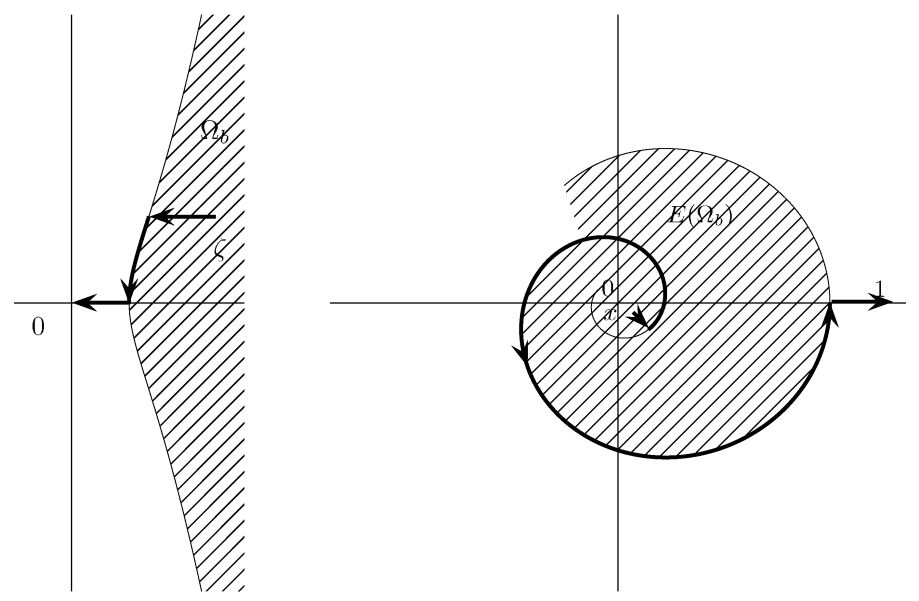

FIGURE 4. Integration path in $\Omega_{b}$ and $E\left(\Omega_{b}\right)$

suffices to show that the length of the path of integration in the $x$ plane is bounded. The radial parts pose no problem, since their length is bounded by 1 . The length of the spiral part is bounded by the total length of the spiral $E\left(\partial \Omega_{b}\right)$, which is calculated in polar coordinates. By direct calculation we see that the modulus $r(\theta)$

of the image by $E$ of $\zeta \in \partial \Omega_{b}$, for $\operatorname{Im}(\zeta) \rightarrow \infty$, is equivalent to $e^{-|\theta|^{\frac{1}{2}}}$. Using the formula for the length element in polar coordinates,

$$
d s=\sqrt{r^{2}(\theta)+r^{\prime 2}(\theta)} d \theta
$$

the boundedness of the length of the spiral follows.

\section{Proofs of the MAIN TheOREMS}

In this section we prove the main theorems, Theorems 1.1 and 1.7

Proof of Theorem 1.7. Let $T$ be the Poincaré time of a hyperbolic polycycle after desingularization and assume that it has a bounded principal part. Note that the Dulac time (contributing to the total Poincaré time) is positive for any vertex of the polycycle. Hence the boundedness assumption on $T$ means that the principal part of the Dulac time of each vertex is bounded. Hence, the Dulac map of each vertex is almost regular. Note also that the time of regular parts is analytic (hence in particular semiregular). The total Poincaré time is calculated according to formula (1.1). By Proposition 2.3, the total Poincaré time $T$ is hence almost regular and by Definition 2.1 it is bounded. The injectivity of the operation $T \mapsto \hat{T}$ of taking the Dulac series now follows from Proposition 2.4

Proof of Theorem 1.1. Let $X, \Gamma, \Sigma$ and $T$ be as in the statement of the theorem. Assume to the contrary that the critical points of the Poincaré time function $T$ accumulate on the polycycle. Then the Dulac series $\hat{T}$ of $T$ is constant (contains just one term which is a constant). But now Theorem 1.7 applies and the Poincaré time is constant. 


\section{ACKNOWLEDGMENT}

We thank J.-P. Rolin for his help.

\section{REFERENCES}

1. C. Chicone; Dumortier, Freddy, Finiteness for critical periods of planar analytic vector fields. Nonlinear Anal. 20 (1993), no. 4, 315-335. MR.1206421 (94b:58082)

2. H. Dulac, Sur les cycles limites, Bull. Soc. Math. France 51 (1923), 45-188. MR1504823

3. J. Écalle, Introduction aux fonctions analysables et preuve constructive de la conjecture de Dulac. (French) [Introduction to analyzable functions and constructive proof of the Dulac conjecture], Actualités Mathématiques. Hermann, Paris, (1992). ii+340 pp. MR 1399559 (97f:58104)

4. J. Guckenheimer; Ph. Holmes, Nonlinear oscillations, dynamical systems, and bifurcations of vector fields. Applied Mathematical Sciences, 42. Springer-Verlag, New York, 1983. xvi+453 pp. MR709768 (85f:58002)

5. Yu. S. Il'yashenko, Limit cycles of polynomial vector fields with nondegenerate singular points on the real plane, Funct. Anal. and Appl., Vol. 18, No. 3, 1984, 199-209. MR757247 (86a:34054)

6. Yu. S. Il'yashenko, Finiteness Theorems for Limit Cycles, Translations of Mathematical Monographs, Volume 94 (1991). MR1109064 (92c:58110)

7. R. Moussu, Le problème de la finitude du nombre de cycles limites. Séminaire Bourbaki, Vol. 1985/86. Astérisque, No. 145-146 (1987), 3, 89-101. MR880027(88g:58159)

8. R. Roussarie, Bifurcation of planar vector fields and Hilbert's sixteenth problem. Progress in Mathematics, 164. Birkhäuser Verlag, Basel, 1998. xviii+204 pp. MR.1628014 (99k:58129)

9. M. Saavedra, Développement asymptotique de la fonction période thèse, Dijon (1995). MR:1298283 (95g:58158)

10. M. Saavedra, Asymptotic expansion of the period function, J. Diff. Eq. 193 (2003), 359-373. MR.1998638 (2004i:34074)

11. M. Saavedra, Asymptotic expansion of the period function II, J. Diff. Eq. 222 (2006), 476-486. MR 2208293

12. E. Titchmarsh. The theory of functions, Second edition. Translated from the English and with a preface by V. A. Rohlin. "Nauka", Moscow, 1980. 464 pp. MR.593142 (82b:30001)

Institut de Mathématique de Bourgogne, U.M.R. 5584 DU C.N.R.S. Université de Bourgogne, B.P. 4787021078 Dijon Cedex, France

E-mail address: mardesic@u-bourgogne.fr

Departamento de Matemática, Facultad de Ciencias Físicas y Matemáticas, UniverSIDAD DE CONCEPCión, CHILE

E-mail address: mariansa@udec.cl 